\title{
Genome Instability of Pseudomonas aeruginosa Phages of the EL Species: Examination of Virulent Mutants
}

\author{
S. V. Krylov ${ }^{a, b}$, E. A. Pleteneva ${ }^{a}$, M. V. Bourkaltseva ${ }^{a}$, O. V. Shaburova ${ }^{a}$, \\ K. A. Miroshnikov ${ }^{b}$, R. Lavigne ${ }^{c}$, A. Cornelissen ${ }^{c}$, and V. N. Krylov ${ }^{a}$ \\ ${ }^{a}$ Federal State Unitary Enterprise of Genetics and Selection of Industrial Microorganisms, Moscow, 117545 Russia; \\ e-mail:krylov@genetika.ru \\ ${ }^{b}$ Shemyakin and Ovchinnikov Institute of Bioorganic Chemistry, Russian Academy of Sciences, Moscow, 119997 Russia; \\ e-mail:kmi@ibch.ru \\ ${ }^{c}$ Laboratory of Gene Technology, Katholieke Universiteit Leuven, Kasteelpark Arenberg 21, B-3001, Leuven, Belgium; \\ e-mail: Rob.Lavigne@biw.kuleuven.be
}

Received March 19, 2010

\begin{abstract}
The article continues a study of pseudolysogeny in Pseudominas aeruginosa infected with phiKZlike phages of the EL species. Analysis was performed for several newly isolated vir mutants of EL phages (EL and RU) that were virulent (capable of causing lysis of bacteria infected with the wild-type phage) and a lower extent of opalescence of negative colonies (NCs). Wile-type recombinants were detected in crosses of virulent mutants of phages EL and RU to confirm the polygenic control of virulence. Since a deletion mutation was found in one of the virulent EL mutants and high genetic instability was characteristic of another mutant, a mobile genetic element was assumed to play a role in mutagenesis. Pseudolysogeny of bacteria provides for horizontal gene transfer between different bacterial strains. Hence, sequencing of the phage genome and demonstration of the lack of toxic gene products are insufficient for the phage to be included into a therapeutic mixture. To use live phages, it is essential to study in detail the possible consequences of their interaction with host bacteria.
\end{abstract}

DOI: $10.1134 / \mathrm{S} 1022795411020116$

\section{INTRODUCTION}

Pseudomonas aeruginosa bacteriophage phiKZ has several unique characters [1-4]. The phages that are phylogenetically related to phiKZ and are active on $P$. aeruginosa belong to several species (phiKZ, Lin68, and EL), which form the phiKZ-like phage genus of the family Myoviridae. Similar phages were revealed only in various species of the genus Pseudominas [512]. It was believed until recently that phiKZ-like phages are virulent, and they were introduced into several commercial mixtures owing to their broad spectrum of lytic activity $[5,6]$. Our results were the first to indicate that infection of phiKZ-like phages may convert the bacterial cell to a specific pseudolysogenic state, when the cell continues divisions for several days to form a colony [7]. However, this state is unstable. Such colonies are lyzed after a while to yield a great amount of the phage, and this is responsible for opalescence of negative colonies (NCs) as an external feature common for $P$. aeruginosa phiKZ-like phages. Detection of phiKZ-like phages in clinical P. aeruginosa isolates supports the idea that pseudolysogeny with these phages arises and is maintained in natural conditions. Previous studies yielded a phage EL mutant that was incapable of causing a pseudolysogenic state and displayed dominance over the wild-type phage, as characteristic of the virulent mutant of usual temperate phages [13]. Here, we describe the properties of new mutant phages of the EL species. The properties suggest an intricate genetic control for the establishment and maintenance of the pseudolysogenic state and support the hypothesis that a certain factor facilitates genetic instability of a phage.

\section{MATERIALS AND METHODS}

We used $P$. aeruginosa strain PAO1, its phage-resistant variants selected previously [14], and clinical $P$. aeruginosa isolates from several burn care centers of Germany, Belgium, and Russia.

We used wild-type bacteriophages of the phiKZlike genus (phiKZ, Lin68, and EL); phage Che21/5, which is related to EL and RU phages; ELvir; and EL, RU, and Che 21/5 mutants similar in NC phenotype [13]. Comparison of phage resistance for bacterial mutants were carried out using various phiKZ-like phages and other phages, including D3, phi297, PMG1, L0, D3112cts, B3cts, B39, TL, 1/1, Yu9, PB1, phi44, OF, and G101, for which resistant $P$. aeruginosa mutants were selected previously [14].

LB agar was used to grow bacteria [15].

Phages were obtained by confluent lysis [16]. 
Phage preparations were purified and concentrated by centrifugation in a $\mathrm{CsCl}$ gradient [17]. Tests for dominance or recessiveness were performed as in [13].

Phage DNA isolation followed a standard protocol [17] or J. Chen's method with minor modification (zchen@aquaplasmid.com, www.aquaplasmid.com). The latter obviates the needs to purify the phage by centrifugation in a $\mathrm{CsCl}$ gradient and to use phenol.

Digestion with restriction endonucleases and agarose gel electrophoresis followed standard protocols [17].

A DNA fragment of phage ELvir1 was sequenced as in [9].

To cross the virulent mutants of different phages of the EL genus (ELvir11 $\times$ RUvir1), the phages were mixed at equal titers, and a drop of the mixture was applied onto a PAO1 lawn. After overnight incubation at $37^{\circ} \mathrm{C}$, material from a lysis plaque was seeded on Petri dishes at a density of approximately $1000 \mathrm{NCs}$ per dish. Recombinant opalescent NCs were easily detectable after incubation at $37^{\circ} \mathrm{C}$ for 2 days.

\section{RESULTS}

\section{Phenotypic Differences of Newly Isolated Mutant Phages of the EL Species}

The mutants isolated differed in the extent of NC opalescence and in virulence, which was determined as the capability to cause lysis of cells infected with the wild-type phage [13]. The extent of virulence was inferred from the lack of opalescence in the overlap of wild-type and mutant phage growth areas (Fig. 1). Phenotypically, the mutants were divided into several groups. Group 1 included the mutants that totally lost NC opalescence (such as ELvir5, ELvir11, and several others). All of these mutants displayed the properties of typical virulent mutants of temperate phages. Group 2 included the mutants that partly preserved NC opalescence and were virulent (e.g., ELvir2 and ELvir10). One of the mutants (ELvir10) gave origin to ELvir $10 \mathrm{~m}$, a secondary mutant that totally lost opalescence and virulence. Group 3 included the mutants that completely lost opalescence and had the extent of virulence only slightly varying among experiments (e.g., ELvir4 and ELvir8). The mutants behaved like group 1 mutants in some cases and were similar to the ELvir10m secondary mutant in some other cases. Group 4 included the ELvir15 mutant, which displayed weak opalescence and weak virulence. The mutant differed from the other mutants and the wildtype phage by its instability. Phenotypically different variants were easily detectable in some cases where individual NCs of the mutant were seeded and incubated for 2-3 days (Fig. 2).

Thus, the extent of virulence did not always correlate with the extent of $\mathrm{NC}$ opalescence in the mutants.

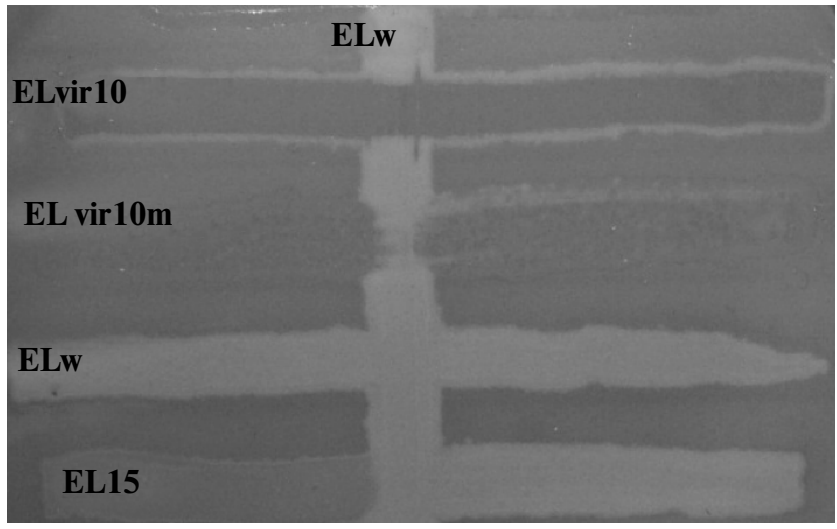

Fig. 1. Testing phage EL mutants for dominance in establishing pseudolysogeny on a $P$. aeruginosa PAO1 lawn. The wild-type phage (ELw) was seeded with a vertical stroke, and mutants were seeded with horizontal strokes. ELvir10 dominates, causing lysis of pseudolysogenic bacteria. EL15 and ELvir10m almost fail to prevent pseudolysogeny.

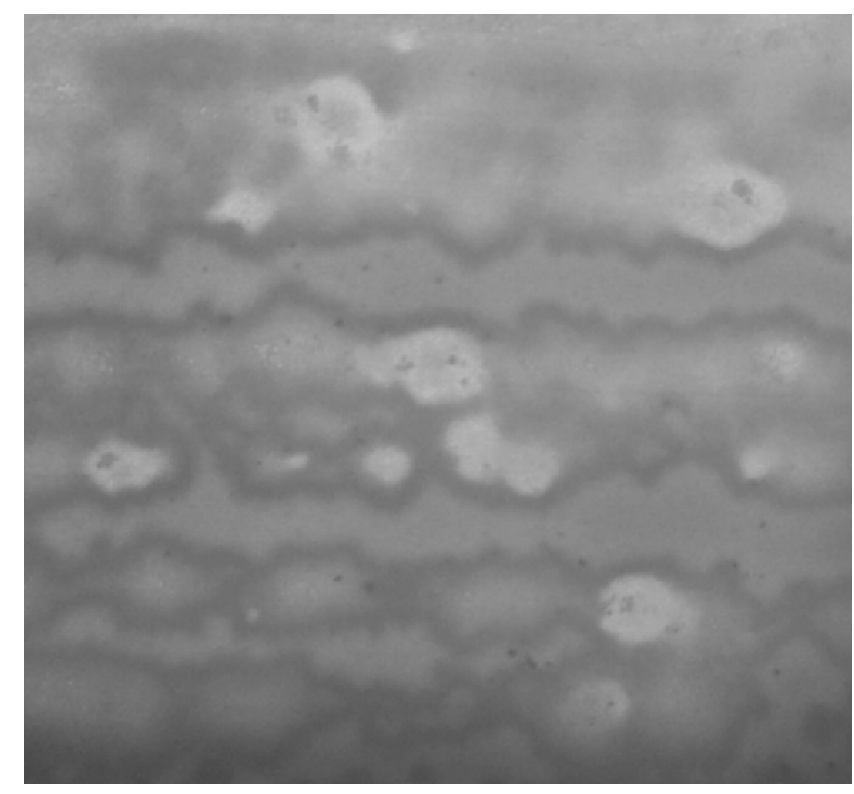

Fig. 2. Instability of the NC morphology in the progeny of the EL15 mutant on a $P$. aeruginosa PAO1 lawn after 3-day incubation.

\section{Restriction Enzyme and Genetic Analyses of the Mutant Phages of the EL Species}

Restriction enzyme analysis of DNA was used to further differentiate the mutants. Restriction profiles obtained with SalI for the wild-type EL phage and several mutants are shown in Fig. 3. A difference from the wild-type phage was observed in this experiment only for group 2 mutants (ELvir2 and ELvir10), which had a small deletion from one of the upper SalI fragments (fragment corresponding to nucleotides $206291 \mathrm{bp}-$ $7305 \mathrm{bp}$ on a circular map and containing open reading frames (ORFs) 194-201 and 1-8) [9]. To check 


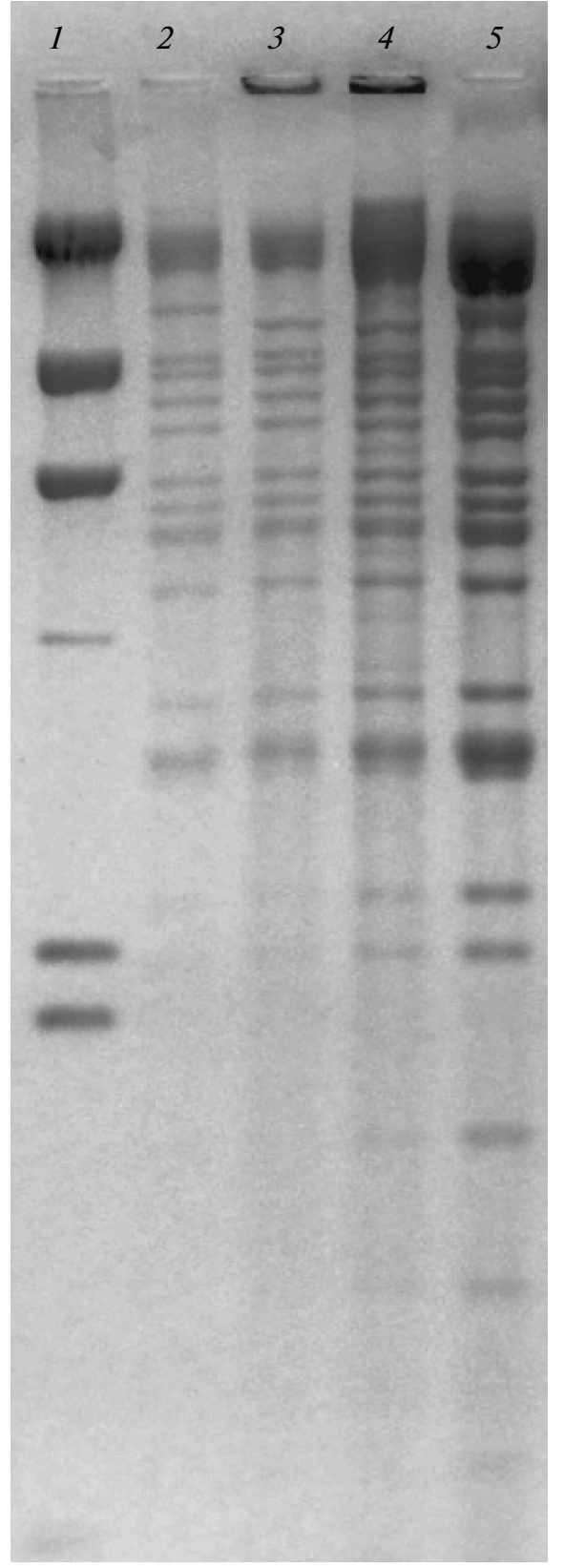

Fig. 3. Digestion with SalI of DNAs of (2) the wild-type phage ELw and its mutants (3) ELvir2, (4) ELvir10, and (5) ELvir5. Lane 1, phage $\lambda /$ HindIII.

whether mutation sites were identical in the phages undistinguishable by DNA restriction enzyme analysis, the phages were crossed. The ELvir5 mutant, which totally lacked opalescence and completely dominated over the wild-type phage, was crossed with several phenotypically similar mutants selected independently of each other. The progeny was tested for the presence of phages whose NCs were similar to wild-type phage NCs in displaying complete opalescence. Three out of five crosses revealed single wildtype recombinants (no more than $3-5$ out of several thousands of NCs of the phage progeny). Although quantitative analysis was unfeasible because of the low sample size, we concluded that the mutations responsible for the similar phenotypic effects in different phages arose in different sites, but, most likely, in one cluster as judged from the low frequency of recombinants. In addition, we crossed virulent mutants of different phages of the EL species (ELvir11 and RUvir1). The two mutants totally lacked opalescence and displayed cross-virulence relative both of the wild-type phages, suggesting their homoimmunity. We seeded the material obtained in the crossing experiment and selected NCs with a high extent of opalescence, characteristic of the wild-type phages. The proportion of such NCs was several times higher than in the above crosses of the EL mutants. DNAs of several EL-RU hybrids were digested with SalI (Fig. 4). The parental mutants did not differ in restriction profile from the original wild-type phages. At the same time, the mutants selected at random proved to differ from both each other and the parental phages. Since the hybrids differed from each other, it is possible to assume that they are a result of two recombination events or that the mutation of phage RUvir1 affected a region other than the above cluster of the EL phages. However, a preliminary sequencing of the phage RU genome is necessary for the exact localization of the mutations in the EL and RU genomes.

\section{Sequencing of the ORF163 Region}

Analysis of the phage EL genome sequence revealed an ORF that has similarity to one of the two phage P22 repressors (mnt), codes for a product similar to the Mnt protein, and has a promoter in its vicinity. We assumed that this region might harbor the mutations that affect the establishment of pseudolysogeny in bacteria. However, sequencing of region 164008-165915, which contains ORF163 and the promoter, did not reveal any difference between the ELvir1 mutant and the wild-type phage.

\section{Effect of the Virulence Mutation on the Lytic Activity Spectrum of Phages of the EL Species}

To check whether a new virulence mutation affected the lytic activity spectrum of the phage, we compared the growth of the virulent mutants, the corresponding wild-type phages, and several other $P$. aeruginosa phages (see Materials and Methods) on two collections of clinical isolates. Pseudomonas aeruginosa clinical isolates greatly vary in the ability to sustain the growth of phages belonging to different species of the phiKZ-like phage genus. Strains selectively sensitive to a limited number of phage species are rather common among $P$. aeruginosa clinical isolates [18]. Some of them sustain the growth of only phiKZ-like phages of the three species. The EL mutant phages did not differ in lytic activity spectrum 
from the corresponding wild-type phages on all clinical isolates examined.

\section{DISCUSSION}

It was long believed that phages with large genomes always display true virulence; i.e., the infected bacterium inevitably dies within the one-step growth cycle of the phage. The possibility of lysogeny was assumed for $P$. aeruginosa infected with phiKZ-like phages on the basis of two observations. First, cells genetically sensitive to the phage still formed colonies in the presence of large amounts of the infecting phage [7]. Second, certain clinical isolates were found to produce phages of the phiKZ or EL species [13]. A pseudotemperate character of a large Clostridium botulinum phage was confirmed by analysis of the inheritance of a converting trait [19]. However, to directly demonstrate a temperate character of phages (including large ones), it is necessary to identify a genetic mechanism similar to the mechanism that controls stable lysogeny in classical temperate phages (a repressor, an operator, etc.).

As a main result of this work, we confirmed that the genomes of EL phages have independent sites whose mutations alter lysogeny and cause various extents of virulence. Lysogeny has a polygenic control, and the corresponding genes seem to be rather far apart because different recombinants form easily in intraspecific crosses of EL and RU phages. The fact that a secondary mutation changed the phenotype of the ELvir10 mutant testifies again to the complexity of the regulatory mechanism. Sequencing of the EL genome allows several assumptions on the origin of this mechanism. The genome of phage EL contains ORF163, whose product is similar to the Mnt repressor, one of the two repressors of moderate phage P22, which is active on Salmonella species. Several properties displayed by phage EL upon pseudolysogeny are highly similar to bacterial lysogeny caused by phage P22. The maintenance of lysogeny in cells carrying the P22 prophage depends on the function of the products encoded by the $c 2$ and $m n t$ genes [20,21], which are in different regions of the P22 genome [22]. The Mnt repressor is involved in regulating the sie (superinfection exclusion) gene [23], and a prophage with a sie mutation fails to exclude a superinfecting phage [24]. The VirA (mapping close to or within the mnt locus) and VirB loci are in different regions of the phage P22 genome [21, 22]. An important feature of cell lysogeny by phage P22 is that the lysogeny rate increases at a higher multiplicity of infection. The cause is that the cell is capable of synthesizing phage DNA only to a limited extent [25]. Phage EL lacks its own DNA polymerase [9], and its replication totally depends on the availability of bacterial replication machinery. It is possible that infected bacteria survive upon multiple infection because there is not enough bacterial DNA polymerase for replication of phage EL DNA. Since pseudolysogeny of $P$. aeruginosa arises upon multiple

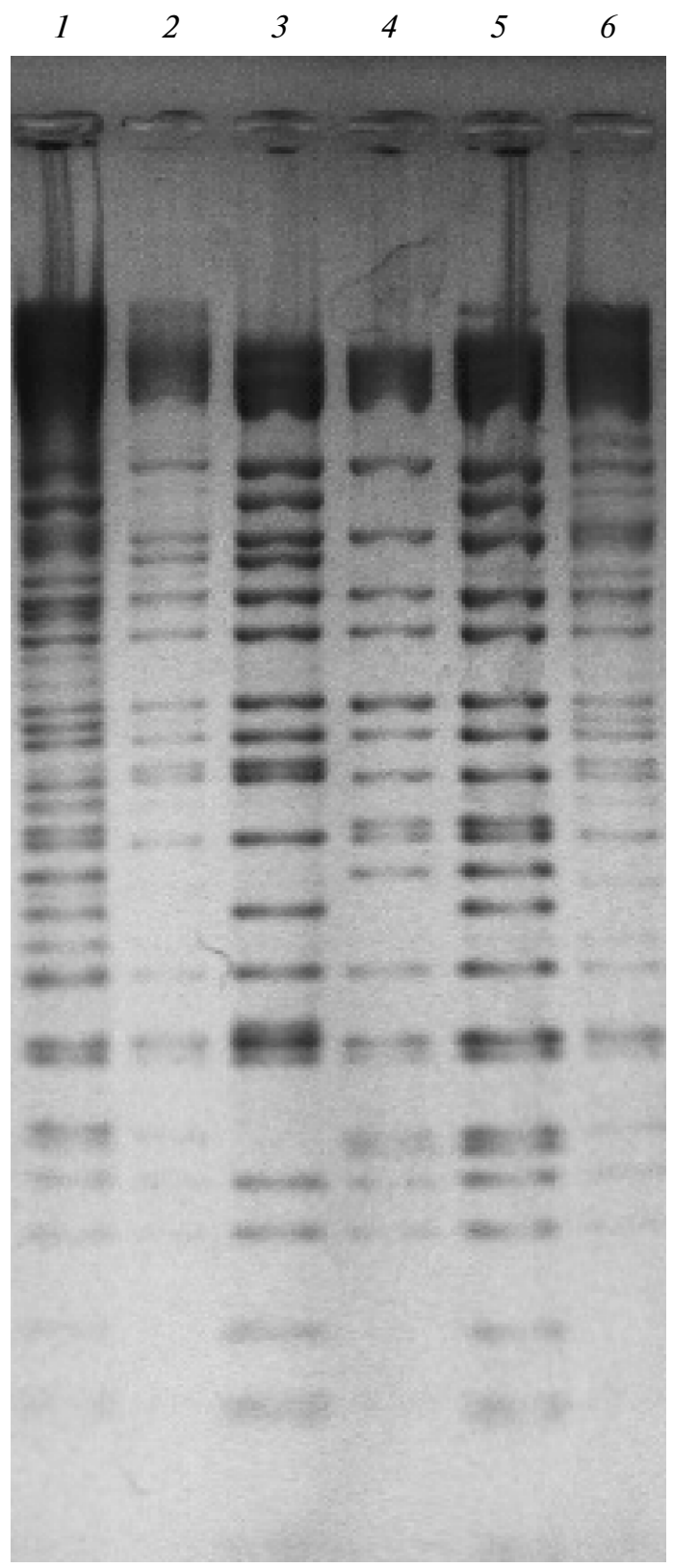

Fig. 4. Digestion with SalI of DNAs of (1) phage RUvir1, (2) phage EL11, and (3-6) EL11-RUvir1 hybrids.

infection with phage EL [13], we assumed that the product of ORF163 of phage EL is involved as a repressor in lysogeny and that virulence mutations arise either within this gene or in its vicinity, as is the case with phage P22. However, sequencing of the corresponding genome region did not reveal any difference from the wild-type phage. Another genome region that harbors lysogeny-controlling genes of phage EL may occur in the region of the deletion found in the ELvir2 mutant. A secondary mutation arose in the ELvir10 genome to totally abolish lysogeny. Hence, it seems promising to further study this EL genome region ( 7305 bp at the beginning and $4323 \mathrm{bp}$ 
at the end of the genome). The appearance of a deletion mutant and instability arising periodically in the ELvir15 mutant suggest a role of a mobile genetic element in the induction of mutations. It is still unclear how the pseudotemperate character of phages of the EL species evolves, i.e., whether the phages evolve to become true temperate phages or a temperate character was inherent in ancestral phages and is to be lost. The genome of the phiKZ phage, which also displays pseudotemperate properties, has a gene for a repressor similar to the repressor of a Bacillus subtilis phage [4]. It is of interest to sequence the genome of phage Lin68, which is the third species of the group and has only minor DNA homology to phage phiKZ [7]. A difference in origin between the repressors of these phages may reflect a difference in their evolution preceding the appearance of the phages in one host, $P$. aeruginosa [26].

Our findings have several applied consequences. First, it is clear that, to include a phage into a therapeutic mixture, it is insufficient to make a positive conclusion on the basis of genome sequencing and product annotation that the phage genome lacks genes whose products are unsuitable for phage therapy. It is necessary to physiologically study the phage-bacterium interactions. Second, since none of the virulent mutants differed in lytic activity spectrum from the corresponding wild-type phages, it seems acceptable to replace the wild-type phiKZ phages in therapeutic mixtures with their virulent mutants. The use of virulent mutants will overcome the problem of pseudolysogeny of infected bacteria and will possibly allow more rapid lysis of the $P$. aeruginosa population in the infection focus. However, generation of a mutant that is incapable of reversion to the wild type is an essential prerequisite to this phage replacement.

\section{ACKNOWLEDGMENTS}

We are grateful to M. Vaneechoutte (Ghent University Hospital, Belgium) and M.A. Popova (Chelyabinsk Clinical Hospital no. 6, Russia) for P. aeruginosa clinical isolates and to J. Chen (www.aquaplasmid.com) for the AquaRNA reagent for rapid isolation of phage DNA.

This work was supported by the Russian Foundation for Basic Research (project no. 08-04-00163-a).

\section{REFERENCES}

1. Krylov, V.N. and Zhazykov, I.Zh., Pseudomonas aeruginosa Bacteriophage phiKZ as a Probable Model for Analysis of Genetic Control of Morphogenesis, Genetika (Moscow), 1978, vol. 14, no. 4, pp. 678-685.

2. Khrenova, E.A., Akhverdyan, V.Z., and Krylov, V.N., Relationship of Pseudomonas aeruginosa Bacteriophages phiKZ and 21 Possessing Unique Nucleoproteid Structure in the Head, Mol. Genet. Mikrobiol. Virusol., 1984 , no. 5, pp. 31-34.
3. Krylov, V.N., Smirnova, T.A., Minenkova, I.B., et al., Pseudomonas Bacteriophage phiKZ Contains an Inner Body in Its Capsid, Can. J. Microbiol., 1984, vol. 30, no. 6, pp. 758-762.

4. Mesyanzhinov, V.V., Robben, J., Grymonprez, B., et al., The Genome of Bacteriophage phiKZ of Pseudomonas aeruginosa, J. Mol. Biol., 2002, vol. 317, pp. 1-19.

5. Sharibzhanova, T.O., Akhverdyan, V.Z., and Krylov, V.N., A Comparative Study of DNA Homology and Morphology of Pseudomonas aeruginosa Bacteriophages to Reveal Phylogenetic Relationships and for an Express Classification, Russ. J. Genet., 1992, vol. 28, no. 3, pp. 24-32.

6. Bourkaltseva, M.V., Sykilinda, N.N., Pleteneva, E.A., et al., Comparisons of the Genomes of New Giant Phages Isolated from Environmental Pseudomonas aeruginosa Strains of Different Regions, Russ. J. Genet., 2004, vol. 40, no. 4, pp. 363-368.

7. Bourkaltseva, M.V., Krylov, V.N., Pleteneva, E.A., et al., Phylogenetic Characterization of a Group of Giant phiKZ-Like Bacteriophages of Pseudomonas aeruginosa, Russ. J. Genet., 2002, vol. 38, no. 11, pp. 1242-1250.

8. Shaburova, O.V., Hertveldt, K., de la Cruz, D.M.A., et al., Comparison of New Giant Bacteriophages OBP and Lu11 of Soil Pseudomonads with Bacteriophages of the phiKZ-Supergroup of Pseudomonas aeruginosa, Russ. J. Genet., 2006, vol. 42, no. 8, pp. 877-885.

9. Hertveldt, K., Lavigne, R., Pleteneva, E., et al., Genome Comparison of Pseudomonas aeruginosa Large Phages, J. Mol. Biol., 2005, vol. 354, no. 3, pp. 536-545.

10. Thomas, J.A., Rolando, M.R., Carroll, C.A., et al., Characterization of Pseudomonas chlororaphis Myovirus 201varphi2-1 via Genomic Sequencing, Mass Spectrometry, and Electron Microscopy, Virology, 2008, vol. 376, no. 2, pp. 330-338.

11. Krylov, V.N., Dela Cruz, D.M., Hertveldt, K., and Ackermann, H.-W., "phiKZ-Like Viruses", a Proposed New Genus of Myovirus Bacteriophages, Arch. Virol., 2007, vol. 152, no. 10, pp. 1955-1959.

12. Krylov, V.N., Miroshnikov, K.A., Krylov, S.V., et al., Interspecies Migration and Evolution of Bacteriophages of the Genus phiKZ: The Purpose and Criteria of the Search for New phiKZ-Like Bacteriophages, Russ. J. Genet., 2010, vol. 46, no. 2, pp. 138-145.

13. Pleteneva, E.A., Krylov, S.V., Shaburova, O.V., et al., Pseudolysogeny of Pseudomonas aeruginosa Bacteria Infected with phiKZ-Like Bacteriophages, Russ. J. Genet., 2010, vol. 46, no. 1, pp. 20-25.

14. Pleteneva, E.A., Shaburova, O.V., and Krylov, V.N., A Formal Scheme of Adsorptional Receptors in Pseudomonas aeruginosa and Possibilities for Its Practical Implementation, Russ. J. Genet., 2009, vol. 45, no. 1 , pp. 35-40.

15. Miller, J.H., Experiments in Molecular Genetics, New York: Cold Spring Harbor Lab., 1972.

16. Adams, M.H., Bacteriophages, New York: Interscience, 1959. 
17. Sambrook, J., Fritsch, E.F., and Maniatis, T., Molecular Cloning: A Laboratory Manual, Cold Spring Harbor: Cold Spring Harbor Lab., 1989.

18. Visca, P., Chiarini, F., Vetriani, C., et al., Epidemiological Typing of Uropathogenic Pseudomonas aeruginosa Strains from Hospitalized Patients, J. Hosp. Infect., 1991, vol. 19, no. 3, pp. 153-165.

19. Sakaguchi, Y., Hayashi, T., Kurokawa, K., et al., The Genome Sequence of Clostridium botulinum Type C Neurotoxin-Converting Phage and the Molecular Mechanisms of Unstable Lysogeny, Proc. Natl. Acad. Sci. USA, 2005, vol. 29, no. 48, pp. 17472-17477.

20. Levine, M. and Smith, H.O., Sequential Gene Action in the Establishment of Lysogeny, Science, 1964, vol. 146, pp. 1581-1582.

21. Bronson, M.J. and Levine, M., Virulent Mutants of Phage P22, Virology, 1972, vol. 47, no. 3, pp. 644-655.
22. Gough, M., Second Locus of Bacteriophage P22 Necessary for the Maintenance of Lysogeny, J. Virol., 1968, vol. 2, no. 10, pp. 992-998.

23. Tokuno, S.I. and Gough, M., Regulation of Bacteriophage P22 DNA Synthesis and Repressor Levels in P22cly Infections, J. Virol., 1977, vol. 21, no. 3, pp. 956-964.

24. Walsh, J. and Meynell, G.G., The Isolation of Nonexcluding Mutants of Phage P22, J. Gen. Virol., 1967, vol. 1, pp. 581-582.

25. Steinberg, B.M. and Gough, M., Bacteriophage P22 Lysogenises Efficiently at High Multiplicities of Infection Because Salmonella typhimurium DNA Synthetic Capacity Is Limited, Nature, 1976, vol. 263, no. 5572, pp. 54-56.

26. Krylov, V., Pleteneva, E., Bourkaltseva, M., et al., Myoviridae Bacteriophages of Pseudomonas aeruginosa: A Long and Complex Evolutionary Pathway, Res. Microbiol., 2003, vol. 154, pp. 269-275. 